\title{
BMJ Open Health outcomes of patients with chronic disease managed with a healthcare kiosk in primary care: protocol for a pilot randomised controlled trial
}

\author{
Grace Ng, ${ }^{1,2}$ Sze Wee Tan, ${ }^{3}$ Ngiap Chuan $\operatorname{Tan}^{1}$
}

To cite: Ng G, Tan SW, Tan NC. Health outcomes of patients with chronic disease managed with a healthcare kiosk in primary care: protocol for a pilot randomised controlled trial. BMJ Open 2018;8:e020265. doi:10.1136/ bmjopen-2017-020265

- Prepublication history and additional material for this paper are available online. To view these files, please visit the journal online (http://dx.doi. org/10.1136/bmjopen-2017020265).

Received 24 October 2017 Revised 5 February 2018 Accepted 15 February 2018
Check for updates

${ }^{1}$ Singapore Health Services (SingHealth) Polyclinics,

Singapore

2Biomedical Research Council, Agency for Science, Technology and Research (A*STAR),

Singapore

${ }^{3}$ Science and Engineering Research Council, Agency for Science, Technology and Research (A*STAR), Singapore

Correspondence to

Dr. Grace $\mathrm{Ng}$;

grace.ng.b.h@singhealth.com. $\mathrm{sg}$

\section{ABSTRACT}

Introduction The rising prevalence of chronic disease is leading to an increase in the demand for primary care services and a shortage of primary care physicians globally. Addressing these challenges calls for innovations in the healthcare delivery model with greater use of healthcare technology tools. We previously examined the feasibility of using an automated healthcare kiosk for the management of patients with stable chronic disease in the primary care setting. The aim of this follow-up study is to evaluate the health outcomes of patients with chronic disease who are on kiosk management compared with patients who are on routine management by nurse clinicians.

Methods and analysis The pilot study will be a twoarmed randomised controlled trial of 120 patients with well-controlled chronic disease on 4-monthly follow-up visits over a 12-month period. Patients with prior diagnoses of hypertension, hyperlipidaemia and/or diabetes will be included in the study and will be randomly assigned to intervention or control groups to receive kiosk or nurse management, respectively. The main primary outcome measure is the overall chronic disease control of the patients. Other primary outcome measures are the blood pressure and low-density lipoprotein cholesterol levels for patients without diabetes, and blood pressure, low-density lipoprotein cholesterol and haemoglobin A1c levels for patients with diabetes. Secondary outcome measures are visit duration, patient satisfaction with the management process, healthrelated quality of life and the occurrence of any adverse event. Data will be captured longitudinally at baseline, 4 months, 8 months and 12 months, and will be analysed using multiple regression models.

Ethics and dissemination The study has been approved by the Singapore Health Services (SingHealth) Centralised Institutional Review Board (2017/2715). Findings of the study will be submitted for publication in peer-reviewed journals and presented at national and international conferences. Trial registration number NCT03274089; Pre-results.

\section{INTRODUCTION}

\section{Background and significance}

The rising burden of chronic disease is leading to an increase in the demand for
Strengths and limitations of this study

- A novel approach using a healthcare kiosk to manage patients with stable chronic disease is examined; the patients are empowered to monitor and review their own health conditions with reduced dependence on healthcare providers.

- Demonstrating comparable health outcomes for patients managed with a healthcare kiosk to the current standard of care will facilitate the adoption of the kiosk into routine clinical practice, allowing for the optimal use of medical resources.

- Randomised controlled trial design minimises the risk of any selection bias.

- Single-centre study design and small sample size may limit the generalisability of the study findings; however, the study will provide the preliminary data required to design a larger multicentre trial with longer-term follow-up.

- Due to the nature of the intervention, this will be an open-label trial; however, objective assessments of the outcome measures will limit any bias.

primary healthcare services and a shortage of primary care physicians. ${ }^{12}$ By 2025, primary care physician shortage of up to 31000 is expected. ${ }^{3}$ Addressing this shortage entails a multiprong approach that includes innovations in healthcare delivery, greater use of healthcare technology and more efficient use of all healthcare professionals on the care team.

In Singapore, primary healthcare is provided by public polyclinics and private medical clinics. ${ }^{4}$ Approximately $20 \%$ of primary healthcare is provided by 20 polyclinics, with the remaining $80 \%$ provided by around 2000 private medical clinics. The chronic disease load is, however, not distributed in corresponding proportions, with almost half $(45 \%)$ of patients with chronic disease managed by the polyclinics. ${ }^{5}$ The 
polyclinics are therefore responsible for a significant proportion of chronic disease care in Singapore. Together with an ageing population and physician shortage, there is a need for a transformation in the practice and delivery of primary care so as to cope with the increasing patient load.

Patients who seek primary healthcare vary in their time and resource requirements. Patients with acute medical conditions or poorly controlled chronic disease typically require more time and attention compared with patients with well-controlled chronic disease. It would therefore be useful if patient care needs can be appropriately stratified so that more time and resources are allocated to those who need them.

At present, patients with chronic disease make regular follow-up visits to their primary care provider for assessments of their chronic disease status and for changes to their medications as necessary. Patients with well-controlled stable chronic disease may go through several visits to their primary care provider in 3-monthly or 4-monthly intervals with little or no change to their medication regimen. In the polyclinics, these patients are co-managed by a nurse clinician under the Nurse Clinician Service (NCS). However, these patients will still have to routinely wait in line with the rest of the polyclinic crowd for a doctor or nurse consultation before getting their regular supply of medications. We saw the possibility of replacing some of these visits with an automated healthcare kiosk, so that these patients can continue on their current medications if their disease condition remained stable, without having to see a doctor or nurse.

We designed and constructed an interactive self-service healthcare kiosk (Self-empowering and Enabling KioskSEEK MyHealthKiosk) that allows patients to measure their physiological parameters (blood pressure, height and weight) in a sequential manner. ${ }^{6}$ The kiosk combines the patients' physiological parameters and their recent laboratory results to classify patients into good, suboptimal or poor-control groups. The kiosk then produces a result slip for the patient with instructions to continue on their current medications (for patients with good disease control), or to see a nurse or doctor for further management (for patients with suboptimal or poor disease control).

Using SEEK, we performed a feasibility study at a polyclinic (SingHealth Polyclinics (SHP)-Bedok). ${ }^{6}$ One hundred patients with stable chronic disease were recruited for the study. These patients used a kiosk in place of seeing a doctor for two subsequent follow-up visits over 9 months. We verified the accuracy of the kiosk and optimised independent kiosk usage with the provision of multilanguage translations in audio and visual forms. Patients and healthcare providers who participated in the study expressed high levels of acceptance and satisfaction with the use of the healthcare kiosk for chronic disease care.

\section{Study aims}

The aim of this follow-up study is to show equivalence of health outcomes for patients managed with the healthcare kiosk compared with the current standard of care. The main primary outcome measure to be compared is the overall chronic disease control of the patients. Other primary outcome measures to be compared are the blood pressure and low-density lipoprotein cholesterol levels for patients without diabetes, and blood pressure, low-density lipoprotein cholesterol and haemoglobin Alc levels for patients with diabetes.

Secondary outcome measures to be compared are (1) visit duration, (2) patient satisfaction with the management process, (3) health-related quality of life and (4) the occurrence of any adverse event.

We hypothesise that patients with well-controlled chronic disease who are managed by the kiosk show equivalent health outcomes, with the benefit of shorter visit time, when compared with patients who are managed by a nurse clinician.

\section{METHODS AND ANALYSIS \\ Study design}

The study will be a randomised controlled equivalence trial conducted in a public primary care polyclinic in Singapore over a duration of 12 months. Figure 1 shows the study design.

The polyclinic (SHP-Punggol) is sited in the northeastern region of Singapore within an urban setting and adjacent to public housing estates. It serves a growing population of about 146640 multiethnic Asian residents. ${ }^{7}$

\section{Study population}

Patients must meet all the eligibility criteria to be enrolled into the study. Eligible patients (1) are between the ages of 21 and 75 years; (2) have at least one chronic medical condition that includes hypertension, hyperlipidaemia and diabetes mellitus; (3) have blood pressure (BP), low-density lipoprotein cholesterol (LDL-C) and haemoglobin A1c (HbA1c) (for diabetics) levels within the recommended targets according to the Ministry of Health $(\mathrm{MOH})$ Singapore clinical practice guidelines for their current and last follow-up visits; and (4) are able to provide informed consent.

Patients with other comorbidities such as ischaemic heart disease and stroke may also be included in the study. We have excluded patients above 75 years of age for this study as the recommended levels for the clinical indicators of BP and HbA1c are less stringent for these patients compared with the general patient population with chronic disease.

Exclusion criteria are patients who (1) have serum creatinine more than $140 \mathrm{mmol} / \mathrm{L}$ (from tests done within the past year), (2) have serum potassium less than $3.5 \mathrm{mmol} / \mathrm{L}$ or more than $5 \mathrm{mmol} / \mathrm{L}$ (from tests done within the past year), (3) have overt proteinuria (urine protein:creatinine ratio more than 0.5 ), (4) were 


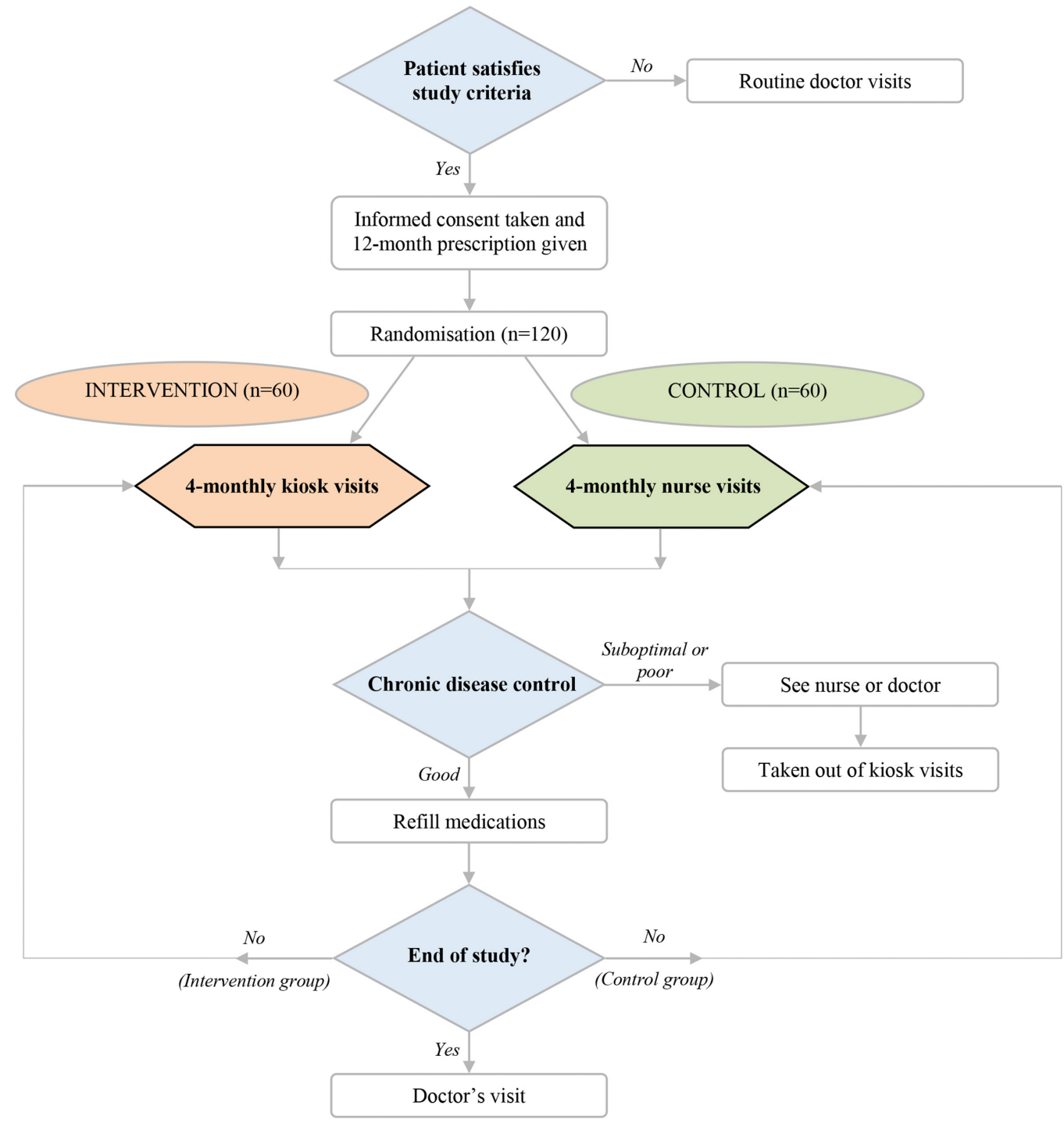

Figure 1 Flowchart of the study protocol.

discharged from hospital or specialist follow-up within the last 6 months, (5) have any pending laboratory or investigation result, (6) are scheduled for any laboratory investigation within the next 4 months (except HbAlc or panel test), (7) have any new complaint related to their chronic medical conditions or (8) have a known history of white coat hypertension.

From our previous feasibility study, ${ }^{6}$ we anticipate the majority of our study patients to be in their fifth to seventh decade of life, to be of Chinese ethnicity and have received secondary school education or less. For this study, the demographic data (age, gender, ethnicity, education level) of all recruited patients will be recorded and analysed.

\section{Sample size estimation}

We have chosen a sample size of 120 based on a realistic estimation of the number of patients that can be recruited from our study site, taking into consideration patient visits, operational constraints and study timeline. The recruitment phase for this study is planned over 80 days.
For our previous study, we recruited one to three patients per day, which gives us an estimated recruitment of 80 to 240 patients.

We acknowledge the small sample size as a limitation. However, the study will serve as a pilot to provide the preliminary data required to design a larger multicentre trial with longer-term follow-up. It will enable us to establish the power calculations and evaluate the financial and logistic feasibility of a full-scale study.

\section{Recruitment and randomisation}

Recruitment for the study will begin in May 2018. Patients will be recruited when they come for their regular chronic care visits at a primary care polyclinic (SHP-Punggol). Eligible patients will be invited by their attending primary care provider to participate in the study. Patients who are agreeable to participate will provide written informed consents to a research coordinator. Patient demographic data (age, gender, ethnicity, education level), disease diagnoses and recent laboratory results will be obtained from the electronic health records (EHRs) of the clinic. 


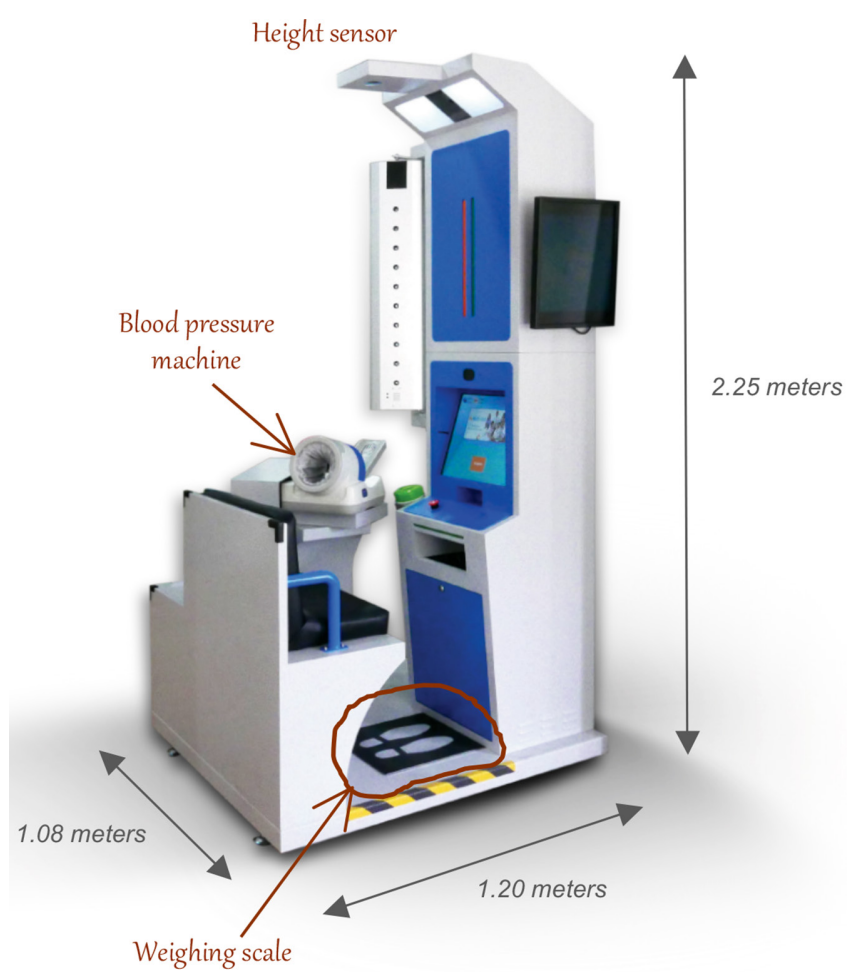

Figure 2 Healthcare kiosk with integrated blood pressure, height and weight measuring devices.

Recruited patients will be given a prescription that will enable them to refill their chronic medications in 4-monthly intervals for the next 12 months and will be randomly assigned to one of two study arms (intervention or control) in a 1:1 ratio (60 patients per arm) by a computerised random-number generator, stratified by diabetic status. Prior to enrolment, group allocation will be concealed in opaque envelopes. Patients in both groups will be reviewed at 4-monthly intervals. To encourage protocol compliance, patients will receive telephone reminders from the research coordinator prior to each follow-up visit. Patients in the intervention group will be evaluated by a healthcare kiosk while patients in the control group will be managed by a nurse clinician. Due to the nature of the intervention, patients will not be blinded to the group allocation. Bias will be minimised by the objective assessments of the outcome measures. All patients will be allowed concomitant medical care during the course of the study.

\section{Study intervention}

We previously designed and developed an interactive self-service healthcare kiosk (SEEK MyHealthKiosk) that is equipped with an automated BP machine, and height and weight measuring devices, which allows patients to measure their own physiological parameters in a sequential manner (figure 2). ${ }^{6}$ The kiosk combines the patients' physiological parameters and recent laboratory results to classify patients into good, suboptimal or poor-control groups. The kiosk then produces a result slip for the patient with instructions to continue on their current medications (for patients with good disease control) or to see a nurse clinician or doctor for further management (for patients with suboptimal or poor disease control, respectively).

The graphical user interface of the kiosk was developed as a web application using Active Server Pages (ASP).NET, with decision algorithms coded in $\mathrm{CH}, \mathrm{C}++$, JavaScript and Hypertext Markup Language. Representative screenshots of the user interface are shown in figure 3.

In this follow-up study, patients who are assigned to the intervention arm will be evaluated by the kiosk at 4 and
A

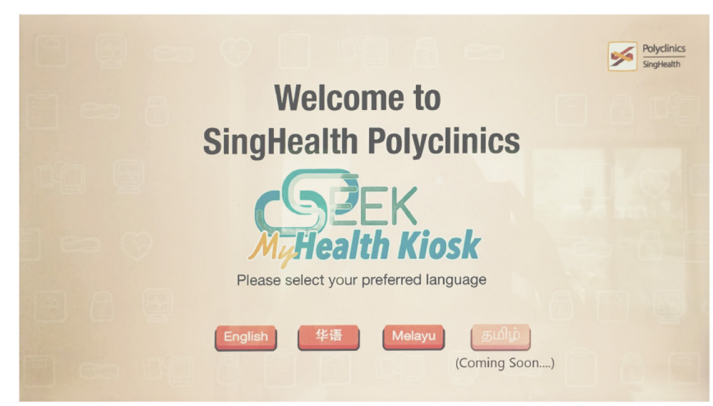

B

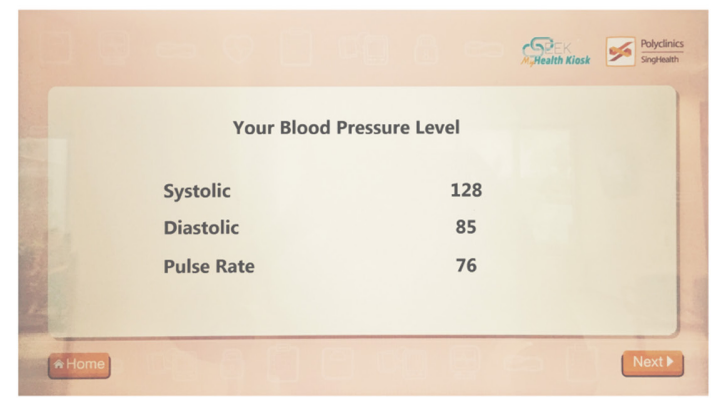

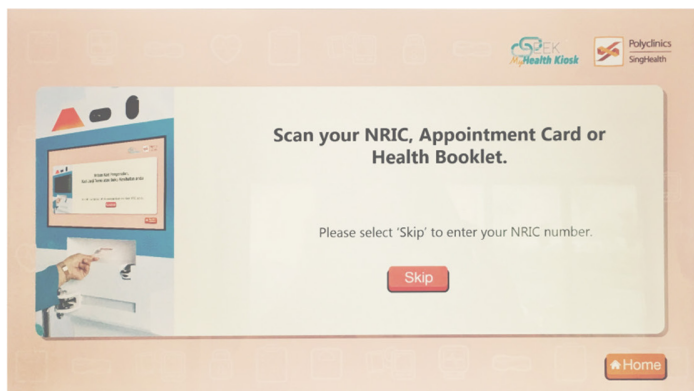

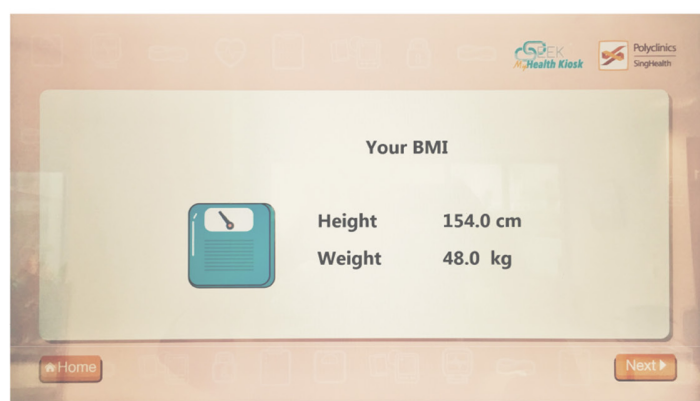

Figure 3 Screenshots of the kiosk graphical user interface: (A) welcome screens, (B) results screens. 
Table 1 Chronic disease risk stratification and disease targets

\begin{tabular}{|c|c|c|c|c|}
\hline Medical conditions & $\begin{array}{l}\text { Ischaemic heart disease } \\
\text { Cerebrovascular accident } \\
\text { (ischaemic) } \\
\text { - Peripheral vascular disease } \\
\text { DM with CKD (stage 3-4) } \\
\text { - Aortic aneurysm } \\
\text { - Familial hypercholesterolaemia }\end{array}$ & $\begin{array}{l}\text { CKD with eGFR }<60 \mathrm{~mL} / \\
\min / 1.73 \mathrm{~m}^{2} \text { or DM (not } \\
\text { both) }\end{array}$ & & \\
\hline Risk stratum & $\begin{array}{l}\text { Very high } \\
\text { (any of the above present) }\end{array}$ & High & Medium & Low \\
\hline \multicolumn{5}{|l|}{ Disease targets } \\
\hline LDL-C (mmol/L) & $<2.1$ & $<2.6$ & $<3.4$ & $<4.1$ \\
\hline Blood pressure $(\mathrm{mm}$ & $<140 / 90$ & $<140 / 90$ & $<140 / 90$ & $<140 / 90$ \\
\hline
\end{tabular}

*By Framingham Risk Score based on age, smoking status, systolic blood pressure, high-density lipoprotein and total cholesterol levels. $\mathrm{CHD}$, coronary heart disease; CKD, chronic kidney disease; DM, diabetes mellitus; eGFR, estimated glomerular filtration rate; HbA1c, haemoglobin A1c; LDL-C, low-density lipoprotein cholesterol.

8 months after recruitment. Patients will interact with the kiosk via a touch screen monitor. Kiosk instructions are available in English, Mandarin and Malay languages in visual and audio forms. Each kiosk interaction begins with scanning of the barcode of the patient's identification card or clinic appointment card, followed by a screening questionnaire to assess if the patient has any acute complaint that requires a doctor's consultation. The patient's blood pressure, height and weight will then be measured. The research coordinator will ensure the patient performs the correct actions and is positioned correctly during physiological measurements.

\section{Kiosk decision algorithm}

Based on their risk-factor profiles (table 1), patients will be stratified into very high, high, medium or low risk categories. Management will involve controlling the disease targets of BP, LDL-C and HbAlc levels according to the prevailing $\mathrm{MOH}$ clinical practice guidelines. ${ }^{8}$

The latest laboratory results (LDL-C and HbAlc (for patients with diabetes)) of recruited patients will be retrieved from the EHR. Based on the answers from the screening questionnaire, combined with the BP measurements and laboratory results, patients will be triaged into well-controlled, suboptimally controlled or poorly controlled groups according to a decision algorithm (table 2).

The overall chronic disease control of a patient will be determined via physiological measurements and laboratory results as described previously. If a patient's chronic disease condition remained well-controlled, the kiosk will advise the patient to continue on his or her current medications. If a patient's chronic disease condition was suboptimally or poorly controlled, the kiosk will advise the patient to consult with a nurse clinician or doctor, respectively. At the end of each kiosk visit, the patient will receive a printout that shows the physiological measurements, laboratory results and kiosk decision (figure 4). The research coordinator will verify the $\mathrm{BP}$ measurement and kiosk decision before allowing the patient to proceed with the kiosk advisory. $\mathrm{BP}$ reading will be verified after each kiosk usage with a repeat measurement taken using a validated automatic digital BP monitor. Kiosk decision accuracy will be verified by checking the patient's parameters and laboratory results against the decision algorithm (table 2) to ensure that the patient has been correctly classified. Kiosk accuracy in terms of triage decisions and BP measurements will be recorded. Patients who have suboptimal or poorly controlled disease will be taken out of further kiosk visits and attend routine clinic consultations for their subsequent follow-up visits.

\section{Control group}

Patients who have been assigned to the control group will be managed by a nurse clinician for their next two visits at 4 and 8 months after recruitment. These patients will be managed as per the standard clinical practice for patients with stable chronic disease on the NCS.

All patients (intervention and control groups) will be reviewed by a doctor at their final study visit 12 months after recruitment.

\section{Withdrawals}

Patients may be withdrawn from the study at any time if (1) the patient makes a voluntary decision to withdraw, (2) the patient has a serious adverse event or develops any medical condition that makes the patient unsuitable for further participation in the study, or (3) the study is terminated. Patients who have withdrawn from the study will continue to receive standard clinical care. 
Table 2 Decision algorithm

For patients with diabetes

Very high risk patient

\begin{tabular}{|c|c|c|c|c|}
\hline Blood pressure $(\mathrm{mm} \mathrm{Hg})$ & $<90 / 60$ & $90 / 60-139 / 79$ & 140/80-159/99 & $\geq 160 / 100$ \\
\hline LDL-C (mmol/L) & & $<2.1$ & $2.1-2.5$ & $\geq 2.6$ \\
\hline HbA1c (\%) & $=6$ & $6-7$ & $7.1-8$ & $>8$ \\
\hline Kiosk decision & $\begin{array}{l}\text { See doctor (any of the } \\
\text { above present) }\end{array}$ & $\begin{array}{l}\text { Medicine refill (all of the } \\
\text { above satisfied) }\end{array}$ & $\begin{array}{l}\text { See nurse (any of the } \\
\text { above present) }\end{array}$ & $\begin{array}{l}\text { See doctor (any of the } \\
\text { above present) }\end{array}$ \\
\hline \multicolumn{5}{|l|}{ High risk patient } \\
\hline Blood pressure $(\mathrm{mm} \mathrm{Hg})$ & $<90 / 60$ & $90 / 60-139 / 79$ & $140 / 80-159 / 99$ & $\geq 160 / 100$ \\
\hline LDL-C (mmol/L) & & $<2.6$ & $2.6-3.3$ & $\geq 3.4$ \\
\hline HbA1c (\%) & $<6$ & $6-7$ & $7.1-8$ & $>8$ \\
\hline Kiosk decision & $\begin{array}{l}\text { See doctor (any of the } \\
\text { above present) }\end{array}$ & $\begin{array}{l}\text { Medicine refill (all of the } \\
\text { above satisfied) }\end{array}$ & $\begin{array}{l}\text { See nurse (any of the } \\
\text { above present) }\end{array}$ & $\begin{array}{l}\text { See doctor (any of the } \\
\text { above present) }\end{array}$ \\
\hline
\end{tabular}

For patients without diabetes

\begin{tabular}{|c|c|c|c|c|}
\hline \multicolumn{5}{|l|}{ Very high risk patient } \\
\hline Blood pressure $(\mathrm{mm} \mathrm{Hg})$ & $=90 / 60$ & $90 / 60-139 / 89$ & $140 / 90-159 / 99$ & $\geq 160 / 100$ \\
\hline LDL-C (mmol/L) & & $<2.1$ & $2.1-2.5$ & $\geq 2.6$ \\
\hline Kiosk decision & $\begin{array}{l}\text { See doctor (any of the } \\
\text { above present) }\end{array}$ & $\begin{array}{l}\text { Medicine refill (all of the } \\
\text { above satisfied) }\end{array}$ & $\begin{array}{l}\text { See nurse (any of the } \\
\text { above present) }\end{array}$ & $\begin{array}{l}\text { See doctor (any of the } \\
\text { above present) }\end{array}$ \\
\hline \multicolumn{5}{|l|}{ High risk patient } \\
\hline Blood pressure (mm Hg) & $90 / 60$ & $90 / 60-139 / 89$ & 140/90-159/99 & $\geq 160 / 100$ \\
\hline LDL-C (mmol/L) & & $<2.6$ & $2.6-3.3$ & $\geq 3.4$ \\
\hline Kiosk decision & $\begin{array}{l}\text { See doctor (any of the } \\
\text { above present) }\end{array}$ & $\begin{array}{l}\text { Medicine refill (all of the } \\
\text { above satisfied) }\end{array}$ & $\begin{array}{l}\text { See nurse (any of the } \\
\text { above present) }\end{array}$ & $\begin{array}{l}\text { See doctor (any of the } \\
\text { above present) }\end{array}$ \\
\hline \multicolumn{5}{|l|}{ Medium risk patient } \\
\hline Blood pressure $(\mathrm{mm} \mathrm{Hg})$ & $=90 / 60$ & $90 / 60-139 / 89$ & $140 / 90-159 / 99$ & $\geq 160 / 100$ \\
\hline LDL-C (mmol/L) & & $<3.4$ & $3.4-4.0$ & $\geq 4.1$ \\
\hline Kiosk decision & $\begin{array}{l}\text { See doctor (any of the } \\
\text { above present) }\end{array}$ & $\begin{array}{l}\text { Medicine refill (all of the } \\
\text { above satisfied) }\end{array}$ & $\begin{array}{l}\text { See nurse (any of the } \\
\text { above present) }\end{array}$ & $\begin{array}{l}\text { See doctor (any of the } \\
\text { above present) }\end{array}$ \\
\hline \multicolumn{5}{|l|}{ Low risk patient } \\
\hline Blood pressure $(\mathrm{mm} \mathrm{Hg})$ & $=90 / 60$ & $90 / 60-139 / 89$ & $140 / 90-159 / 99$ & $\geq 160 / 100$ \\
\hline LDL-C (mmol/L) & & $<4.1$ & $4.1-4.8$ & $\geq 4.9$ \\
\hline Kiosk decision & $\begin{array}{l}\text { See doctor (any of the } \\
\text { above present) }\end{array}$ & $\begin{array}{l}\text { Medicine refill (all of the } \\
\text { above satisfied) }\end{array}$ & $\begin{array}{l}\text { See nurse (any of the } \\
\text { above present) }\end{array}$ & $\begin{array}{l}\text { See doctor (any of the } \\
\text { above present) }\end{array}$ \\
\hline
\end{tabular}

Green (well-controlled) - continue current medications; orange (suboptimally controlled)-see nurse clinician; red (poorly controlled)-see doctor.

HbA1c, haemoglobin A1c; LDL-C, low-density lipoprotein cholesterol.

\section{Study outcomes}

The main primary outcome measure to be compared is the overall chronic disease control of the patients. Other primary outcome measures to be compared are BP and LDL-C for patients without diabetes, and BP, LDL-C and HbAlc for patients with diabetes. All variables except LDL-C will be assessed at baseline, 4 months, 8 months and 12 months post-recruitment. LDL-C levels will be measured from fasting blood samples taken at baseline and 12 months post-recruitment, in accordance to the MOH Singapore clinical practice guidelines that recommend yearly review of LDL-C for patients with stable disease.
The overall chronic disease control (well-controlled, suboptimally controlled, poorly controlled) of all patients will be determined using the decision algorithm (table 2).

For patients in the intervention group, BP will be taken at the kiosk by an integrated automatic BP machine. Measuring devices in the kiosk have been certified by the Health Sciences Authority of Singapore to be of an acceptable standard in accordance to the Health Products Act and Health Products (Medical Devices) Regulations.

For patients in the control group, BP will be taken using a validated automatic digital $\mathrm{BP}$ monitor by a nurse clinician who will adhere to the American Heart Association guidelines for BP measurements. ${ }^{9}$ At each 
A

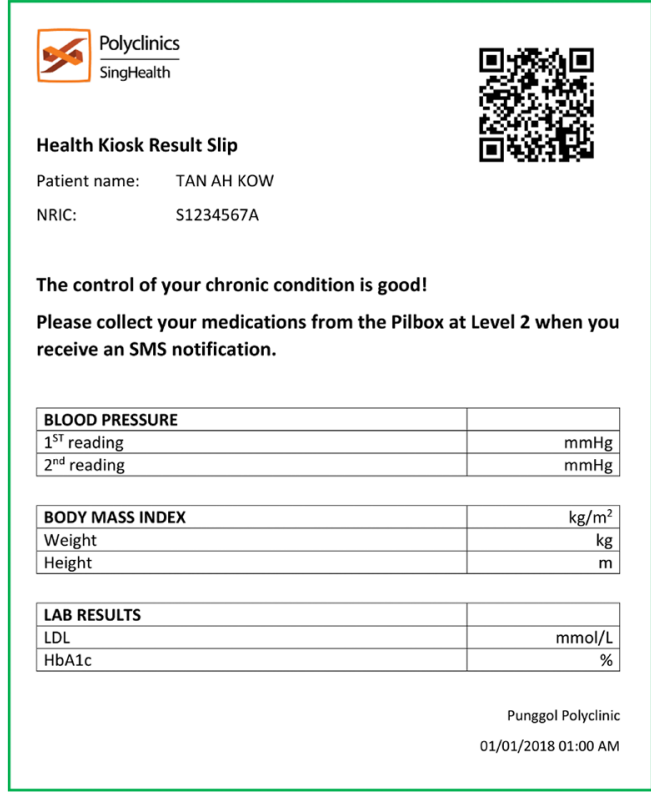

B

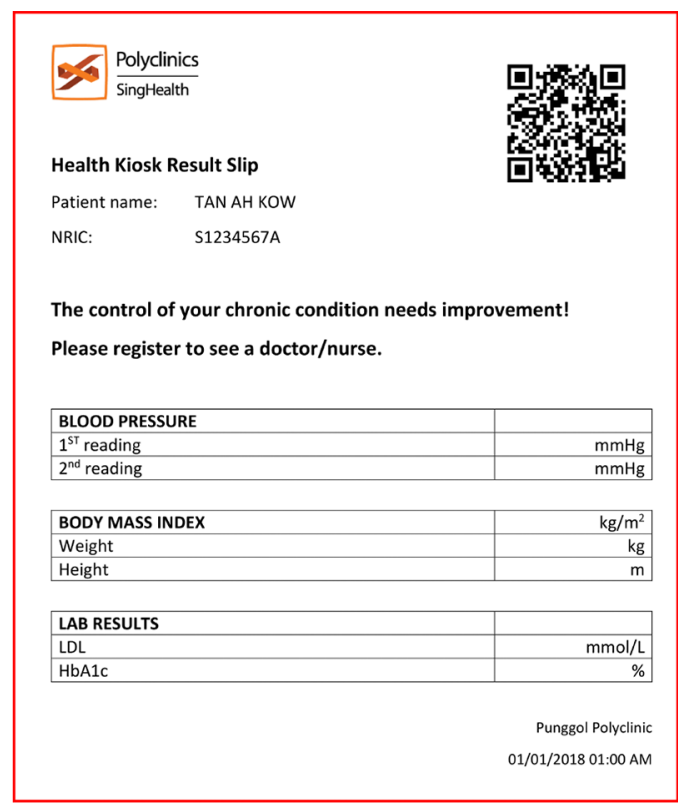

Figure 4 Result slips: (A) good disease control, (B) suboptimal/poor disease control.

visit, the patient's $\mathrm{BP}$ will be established by a minimum of two measurements taken with at least 1 min interval between them, and the average of these readings will be recorded. If there is a greater than $5 \mathrm{~mm} \mathrm{Hg}$ difference between the first and second readings, additional readings will be obtained and the average of the closest readings will be used to represent the patient's BP.

For patients with diabetes, HbA1c will be measured at an on-site laboratory in the clinic. Capillary blood samples will be obtained via finger-pricks done by trained laboratory technicians as per routine clinical practice, and analysed using a point-of-care device, with results available in $6 \mathrm{~min}$. This test will be done just prior to each kiosk usage or nurse consultation, for patients in the intervention and control groups, respectively.

The secondary outcome measures to be assessed are visit duration, patient satisfaction with the management process, health-related quality of life and the occurrence of any adverse event. Data for these variables will be collected at specific time points during the course of the study (table 3).

The duration for each patient visit will be measured from time of registration to end of kiosk usage or nurse consultation, and will be recorded by the research coordinator using a stopwatch.

Patient satisfaction and occurrence of adverse events will be assessed by a questionnaire designed for this study (online supplementary appendix A). Satisfaction with kiosk or nurse management will be assessed on a Likert scale in the following areas: convenience of visit schedule, location of kiosk or nurse consultation, appropriateness of management, duration of time spent at kiosk usage or nurse consultation, and the replacement of a doctor's visit by the kiosk or nurse clinician. Qualitative feedback will be captured via free-text inputs.

Health-related quality of life will be assessed using the EQ-5D-5L instrument (online supplementary appendix B). ${ }^{10}$

\section{Table 3 Data collection schedule}

\begin{tabular}{|c|c|c|c|c|}
\hline Data collected & Recruitment & 4 months & 8 months & 12 months (end of study) \\
\hline Overall disease control & $x$ & $x$ & $x$ & $x$ \\
\hline LDL-C & $x$ & & & $\times$ \\
\hline $\mathrm{HbA1c}$ & $x$ & $x$ & $x$ & $x$ \\
\hline Visit duration & & $x$ & $x$ & \\
\hline $\begin{array}{l}\text { Satisfaction and adverse events } \\
\text { questionnaire }\end{array}$ & & & & $x$ \\
\hline EQ-5D-5L questionnaire & $x$ & & & $x$ \\
\hline
\end{tabular}

HbA1c, haemoglobin A1c; LDL-C, low-density lipoprotein cholesterol. 


\section{Data management and monitoring}

Data collected from the study will be stored electronically and as hardcopies. Electronic data will be stored in a password-protected computer or a thumb drive that is kept in a locked drawer in the principal investigator's (PI's) office, and hardcopy data will be stored in locked cabinets in the PI's office. Only study investigators will have access to the study data.

During the course of the study, the PI will ensure protocol adherence and will review collected data regularly. The PI and research coordinator will check data collection forms fortnightly for completeness and accuracy (including range checks, missing data and double entry). Physical and digital records will be cross-checked for consistency. A research executive from the SHP Department of Research will audit every participant's records at 3-monthly intervals to ensure that record-keeping meets regulatory requirements and is kept up to date.

Data safety and monitoring are subject to audit by the SHP Department of Research and the SingHealth Centralised Institutional Review Board (CIRB). The PI will report any adverse event or deviation from the study protocol to the CIRB within the stipulated timeframe.

An independent trial advisory committee, comprising representatives from the Departments of Clinical Services and Operations, Finance, Nursing, Pharmacy and Information Technology (Integrated Health Information Systems), will be updated on trial progress at quarterly intervals and will give recommendations for discontinuation, modification or continuation of the study. Members of this committee will not be directly involved in the trial and will be free to review any information or study process related to the trial.

\section{Analysis}

\section{Descriptive analysis}

Descriptive statistics will be used to summarise the baseline characteristics of the patients in the study arms. Continuous data will be described in terms of means and SD (or median and IQR for asymmetrical data). Categorical data will be described in terms of percentages.

\section{Statistical tests}

Analysis will be done using SPSS (Statistical Package for Social Sciences) Statistics V.24.0 with an alpha of 0.05 set a priori. The primary outcome variables of overall chronic disease control, BP, LDL-C and HbAlc (for diabetics) will be captured longitudinally over 12 months and will be assessed using multiple regression models (linear and logistic regression analysis for continuous and categorical outcome variables, respectively). The effect of the intervention will be analysed after adjusting for patient age, gender, ethnicity and education level. For the analysis over time, mixed-design analysis of variance will be used, with intervention as the between-subject factor and time as the within-subject factor. The analyses for BP, LDL-C and HbA1c will be applied to the relevant stratified samples.
For demographic and secondary outcome data, continuous variables will be compared using Student's t-test or the Wilcoxon-Mann-Whitney $U$ test, and categorical variables will be compared using Pearson's $\chi^{2}$ test. Data analyses will be performed on an intention-to-treat basis.

\section{ETHICS AND DISSEMINATION}

Research ethics approval

The study will be conducted in accordance to the ethical principles of the Helsinki Declaration guidelines. ${ }^{11}$ Ethical approval for the study has been obtained from the SingHealth CIRB and the study has been registered with ClinicalTrials.gov (NCT03274089). In the event of any important modification to the study protocol, the CIRB, trial advisory committee, study team members and trial participants will be informed. The CIRB will be informed of any protocol change via an online CIRB Amendment Form and any change will be implemented only after approval has been obtained. The trial advisory committee and study team members will be informed of any change to the protocol via email or in person, and trial participants will be informed of any change to the protocol via phone or in person.

\section{Consent}

Patients who are eligible for the study will be referred by their attending primary care provider to the research coordinator who will carry out the informed consent process. Details of the study will be explained to the patients, including its objectives, process, duration, and possible risks and benefits. Patients will be informed that participation in the study is entirely voluntary and their decision to participate in the study will not affect their future medical care. Recruited patients will have the right to withdraw from the study at any time. Patients will be given the opportunity to ask questions about the study and given time for consideration to participate. Patients who decide to participate in the study may then voluntarily sign on the informed consent form (online supplementary appendix $\mathrm{G}$ ) .

\section{Confidentiality}

Recruited patients will be assigned anonymous patient recruitment numbers and all subsequent references and analyses will be performed on de-identified datasets. Patient information collected during the course of the study will be kept confidential in compliance to the Singapore Personal Data Protection Act. All copies of electronic data will be stored in a password-protected computer or thumb drive, and paper documentation will be kept in secure cabinets with access available only to study investigators. All study data will be kept for 6 years.

\section{Declaration of interests}

None of the authors have any financial interest or have received paid compensation for this work. No competing interest that may inappropriately influence this work is 
declared. AIT Technologies Private Limited, which fabricated the kiosk, had no role in the study design, and will have no role in its conduct, data collection, interpretation and analysis.

\section{Dissemination of study findings}

The findings of this study will be submitted for publication in peer-reviewed journals and presented at national and international conferences.

\section{DISCUSSION AND CONCLUSION}

Population ageing and the burden of chronic disease continue to weigh on already overstretched primary care systems worldwide. ${ }^{12}$ By 2025, a primary care workforce crisis is anticipated in the USA. ${ }^{3}$ Strategies to mitigate this shortfall have included innovations in the primary care delivery model, by expanding who provides care and how care is coordinated. ${ }^{13}$ The patient-centred medical home delivers care using a team of healthcare providers comprising physicians, nurse clinicians, pharmacists, social workers and care coordinators, while nurse-managed health centres have nurse practitioners as the primary care providers. ${ }^{1415}$ Advancements in e-health technologies have further enhanced the potential for patient self-care and management. ${ }^{16} 17$

Population growth and ageing presents a combined challenge to the healthcare system in Singapore. ${ }^{18}$ The past decade saw a population growth of $25 \%$, with continued growth expected in the coming years. By 2030, $20 \%$ of the population will be aged 65 years and older. There has been a shift in the focus of healthcare - from episodic care in the acute hospitals to chronic care in the community, which is reflective of the evolving patient profile and their related needs.

In Singapore, the primary care centres comprising public polyclinics and private medical clinics are where most patients receive care for their chronic health conditions. ${ }^{5}$ A substantial proportion $(45 \%)$ of patients with chronic disease receives care from the polyclinics due to the availability of subsidies. However, because only about $14 \%$ of all primary care physicians in Singapore practice in the polyclinics, there is a mismatch between the supply of primary care physicians and the demand for chronic disease care. The ramifications of population ageing and the resultant chronic disease epidemic are manifest in the polyclinics where overcrowding and long waiting times are commonplace. Measures to address this challenge have included efforts to enlarge the primary care workforce, extension of chronic disease healthcare subsidies to the private clinics and initiatives to promote the stratification and right-siting of patient care needs. ${ }^{18} 19$

The establishment of the NCS was taken as a step towards the right-siting of patients with chronic disease in the polyclinics. Only patients with stable chronic disease are managed under the NCS, where they receive shared care from physicians and nurse clinicians. These patients make alternate visits between physicians and nurse clinicians in 4-monthly to 6-monthly intervals, effectively reducing the number of physician visits by half.

For some years now, healthcare kiosks have played a role in streamlining services in the healthcare industry. They are used for patient registration, bill payment and navigation. In recent years, healthcare kiosks have evolved widely. The new generation of healthcare kiosks come integrated with a variety of medical devices that allow for physiological parameters such as blood pressure, height and weight to be measured. ${ }^{20}{ }^{21}$ They are interactive and can provide health information and advice. Undoubtedly, the self-service capacity afforded by healthcare kiosks can provide the leverage to relieve the manpower constraints of our primary care system.

In our current care model, the management of patients with well-controlled chronic disease is shared between physicians and nurse clinicians (figure 5). Incorporating a healthcare kiosk into the care delivery model will facilitate a shift in the spectrum of care, so that the management of these patients is shared between a physician and kiosk, hence freeing nurse clinicians to take on a share in the management of patients with suboptimally controlled disease. Typically, patients with suboptimally controlled disease are initially counselled by nurse clinicians on lifestyle modifications before medication titration by physicians, thus making such a care model intuitive. By having the care of patients with well-controlled and suboptimally controlled disease shared among physicians, nurse clinicians and healthcare kiosks, physician visits for these patients will be reduced, allowing for more physician time to be allocated to the management of patients with poorly controlled disease.

In 2015, we developed a self-service healthcare kiosk and explored the feasibility of using the kiosk for the management of patients with well-controlled chronic disease. ${ }^{6}$ Both patients and physicians who participated in the study expressed high levels of acceptance and satisfaction with using the kiosk for chronic disease care. While previous studies have shown that substituting physicians with nurse clinicians in the healthcare delivery model resulted in equivalent patient outcomes, the impact of using a healthcare kiosk for care delivery is unknown. ${ }^{22-25}$ This follow-up study will assess the health outcomes of patients managed with a healthcare kiosk. It evaluates the maintenance of stable chronic disease for such patients and examines the secondary outcomes of health-related quality of life and adverse events. The findings from the study will help determine if adoption of a healthcare kiosk into the primary care delivery model is a viable option.

The strengths of this study are that it explores a novel approach in using a healthcare kiosk to manage patients with stable chronic disease and uses a randomised controlled trial design to demonstrate comparable health outcomes to the current standard of care. The adoption of the kiosk into routine clinical practice will facilitate optimal resource allocation for patients with varying medical needs. 
Current model of care

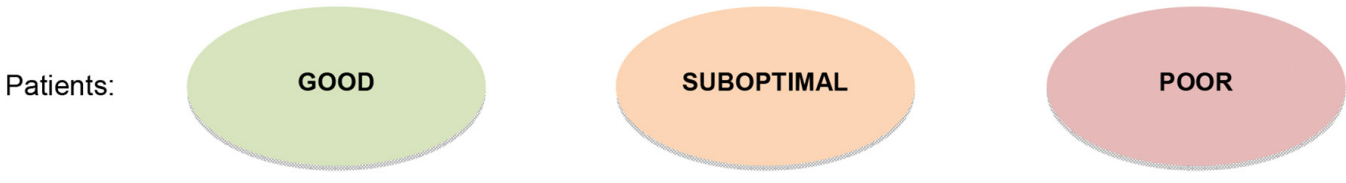

Care spectrum:

Doctor

Nurse

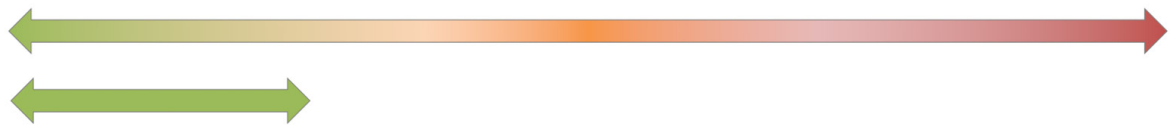

Proposed future model of care

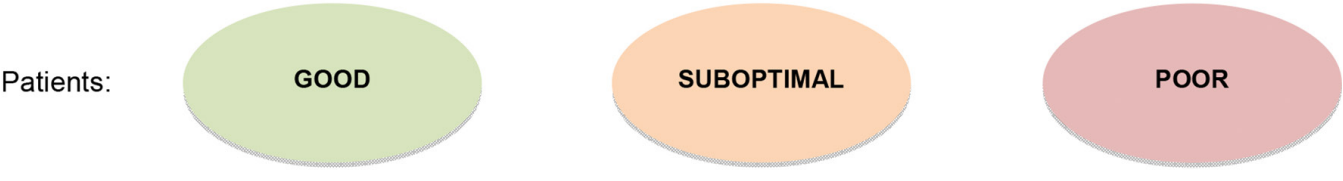

Care spectrum:

Doctor

Nurse

Kiosk

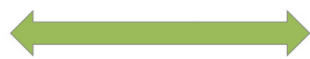

Figure 5 Evolution of the primary care model.

The limitations of this study are its single-centre design, modest sample size and short follow-up duration. The findings will nevertheless provide the preliminary data needed to design a larger multicentre trial with longerterm follow-up.

As we are exploring a new model of care delivery, patients in the study are guided during kiosk usage. With regular use over time, however, we anticipate greater patient confidence and autonomy, as was observed in our feasibility study. ${ }^{6}$ The eventual aim is for the deployment of a self-service healthcare kiosk that will enable its full benefits to be realised in terms of patient empowerment and resource allocation. These may be better appreciated in a longer-term follow-up study.

Patients selected for the study are constrained by age, disease diagnoses and control, and wider scopes shall be considered in subsequent studies. Future studies may explore the management of more elderly patients who are an important class of patients with chronic disease. We may also consider the management of patients with other disease conditions such as hypothyroidism, hyperthyroidism and asthma. The co-management of patients with suboptimally controlled disease may also be examined. The potential integration of other point-of-care devices can further enhance the self-service capacity of the kiosk.

Beyond health outcomes, it is important to assess the cost-effectiveness of a healthcare kiosk in clinical care. Future studies may consider this, each from the perspective of the healthcare organisation and the patient. For the healthcare organisation, the cost of technology versus medical manpower may be evaluated over time, following the establishment of a sustainable payment model for the kiosk. For the patient, the analysis may include the cost benefits derived from reduced physician visits and the flexibility of alternative health service options.

Acknowledgements The kiosk was designed and fabricated by AIT Technologies Private Limited.

Contributors GN designed and conceptualised the study, drafted the manuscript and will analyse the results. TSW and TNC contributed to the study design and revision of the manuscript. All authors have read and approved the final version of the manuscript and agree to be accountable for all aspects of the work in ensuring that questions related to the accuracy or integrity of any part of the work are appropriately investigated and resolved.

Funding This study is supported by an Agency for Science, Technology and Research (A*STAR) grant 1629027001.

Competing interests None declared.

Patient consent Not required.

Ethics approval SingHealth Centralised Institutional Review Board.

Provenance and peer review Not commissioned; externally peer reviewed.

Open Access This is an Open Access article distributed in accordance with the Creative Commons Attribution Non Commercial (CC BY-NC 4.0) license, which permits others to distribute, remix, adapt, build upon this work non-commercially, and license their derivative works on different terms, provided the original work is properly cited and the use is non-commercial. See: http://creativecommons.org/licenses/by-nc/4.0/ 
(c) Article author(s) (or their employer(s) unless otherwise stated in the text of the article) 2018. All rights reserved. No commercial use is permitted unless otherwise expressly granted.

\section{REFERENCES}

1. World Health Organization. The World Health Report 2002: reducing risks, promoting healthy life. 2002 http://www.who.int/whr/2002/en/ (accessed 5 Feb 2018).

2 Goodman DC, Fisher ES. Physician workforce crisis? Wrong diagnosis, wrong prescription. N Engl J Med 2008;358:1658-61.

3. IHS Inc. The complexities of physician supply and demand: projections from 2013 to 2025. Prepared for the Association of American Medical Colleges. Washington, DC: Association of American Medical Colleges, 2015. https://www.aamc.org/ download/426242/data/ihsreportdownload.pdf?cm_mmc=AAMC-_ScientificAffairs-_-PDF-_-ihsreport (accessed 5 Feb 2018).

4 Khoo HS, Lim YW, Vrijhoef HJ. Primary healthcare system and practice characteristics in Singapore. Asia Pac Fam Med 2014;13:8.

5 Sng QS. Ministry of Health Singapore: Primary Care Survey 2010profile of primary care patients. 2010 https://www.moh.gov.sg/ content/moh_web/home/Publications/information_papers/2011/pri mary_care_survey2010profileofprimarycarepatients.html (accessed 5 Feb 2018).

$6 \mathrm{Ng} \mathrm{G}$, Tan N, Bahadin J, et al. Development of an automated healthcare kiosk for the management of chronic disease patients in the primary care setting. J Med Syst 2016;40:169.

7. Singapore Department of Statistics. Population trends. 2017 http://www.singstat.gov.sg/publications/publications-and-papers/ population-and-population-structure/population-trends (accessed 5 Feb 2018).

8. Ministry of Health, Singapore. Clinical practice guidelines: diabetes mellitus. 2014 https://www.moh.gov.sg/content/moh_web/ healthprofessionalsportal/doctors/guidelines/cpg_medical/2014/c pgmed_diabetes_mellitus.html (accessed 5 Feb 2018).

9. Pickering TG, Hall JE, Appel LJ, et al. Recommendations for blood pressure measurement in humans and experimental animals: part 1: blood pressure measurement in humans: a statement for professionals from the Subcommittee of Professional and Public Education of the American Heart Association Council on High Blood Pressure Research. Circulation 2005;111:697-716.

10. Herdman M, Gudex C, Lloyd A, et al. Development and preliminary testing of the new five-level version of EQ-5D (EQ-5D-5L). Qual Life Res 2011;20:1727-36.
11. World Medical Association Declaration of Helsinki. Ethical principles for medical research involving human subjects. JAMA 2013;310:2191-4.

12. Bodenheimer T, Pham HH. Primary care: current problems and proposed solutions. Health Aff 2010;29:799-805

13. Margolius D, Bodenheimer T. Transforming primary care: from past practice to the practice of the future. Health Aff 2010;29:779-84.

14. American Academy of Family Physicians, American Academy of Pediatrics, American College of Physicians, American Osteopathic Association. Joint principles of the patient-centered medical home. 2007 http://www.aafp.org/dam/AAFP/documents/practice_ management/pcmh/initiatives/PCMHJoint.pdf (accessed 5 Feb 2018).

15. Pilon BA, Ketel C, Davidson HA, et al. Evidence-guided integration of interprofessional collaborative practice into nurse managed health centers. J Prof Nurs 2015;31:340-50.

16. Blumenthal D, Glaser JP. Information technology comes to medicine. N Engl J Med 2007;356:2527-34.

17. Silva BM, Rodrigues JJ, de la Torre Díez I, et al. Mobile-health: a review of current state in 2015. J Biomed Inform 2015;56:265-72.

18. Ministry of Health (MOH), Singapore. MOH 2012 Committee of Supply Speech Healthcare 2020: Improving Accessibility, Quality and Affordability for Tomorrow's Challenges (Part 1 of 2). 2012 http:// www.moh.gov.sg/content/moh_web/home/pressRoom/speeches_d /2012/moh_2012_committeeofsupplyspeechhealthcare2020imp rovingaccessibi.html (accessed 5 Feb 2018).

19. Tan NC. PAIR UP for primary care excellence: perspectives from a primary healthcare provider in Singapore. Singapore Med $J$ 2014:55:110-6.

20. Medex Spot Unmanned Micro Clinic. https:// directprimarycarejournal.com/2016/10/08/technology-the-medexspot-unmanned-micro-clinic-nurse-practitioners-at-medex-spotterminal/ (accessed 5 Feb 2018).

21. Higi station. https://higi.com (accessed 5 Feb 2018).

22. Laurant M, Reeves D, Hermens R, et al. Substitution of doctors by nurses in primary care. Cochrane Database Syst Rev 2005;2:CD001271.

23. Martin-Misener R, Harbman P, Donald F, et al. Cost-effectiveness of nurse practitioners in primary and specialised ambulatory care: systematic review. BMJ Open 2015;5:e007167.

24. Martínez-González NA, Tandjung R, Djalali S, et al. Effects of physician-nurse substitution on clinical parameters: a systematic review and meta-analysis. PLoS One 2014;9:e89181.

25. Swan M, Ferguson S, Chang A, et al. Quality of primary care by advanced practice nurses: a systematic review. Int J Qual Health Care 2015;27:396-404. 